\title{
CONDUCTION OF HEAT IN COMPOSITE SLABS*
}

\author{
BY \\ J. C. JAEGER \\ University of Tasmania
}

1. Introduction. Among the most important practical problems in Conduction of Heat are those of flow of heat through composite slabs of several layers with different thermal properties such as occur in the walls of furnaces, rooms, and refrigerated chambers. The well known result for steady flow of heat in such a region is very simple: if $l_{r}, k_{r}$, and $R_{r}=l_{r} / k_{r}$ are the thickness, thermal conductivity, and thermal resistance, respectively, of the $r$-th layer, the rate of flow of heat, per unit time, per unit area, per unit temperature difference, through a wall of $n$ such layers with perfect thermal contact between them is

$$
\left\{R_{1}+R_{2}+\cdots+R_{n}\right\}^{-1},
$$

and the same result holds if some of the layers consist of air spaces or surface films of negligible heat capacity provided each film is represented by an appropriate thermal resistance.

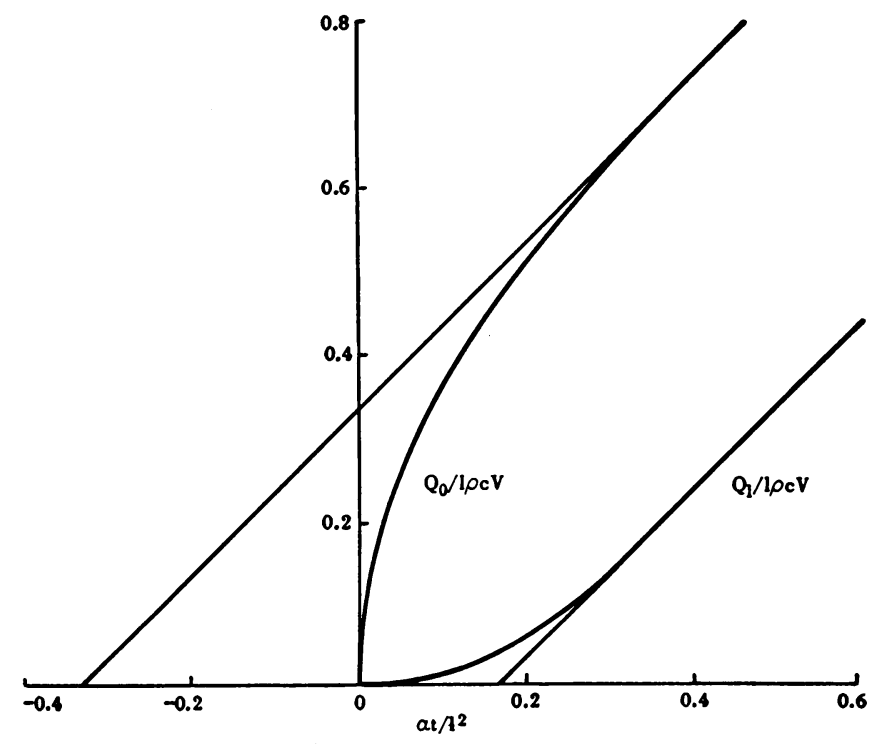

FIG. 1.

In contrast to the simplicity of (1) the complete solutions of transient problems on composite walls, ${ }^{1}$ even in the simplest case of two layers only, are so very complicated as to be practically useless. Information is needed about many transient problems such as the warming up of a furnace, the initial cooling of a refrigerated chamber, and the

*Received April 19, 1949.

'For references and some solutions see Carslaw and Jaeger, Conduction of heat in solids, Oxford, 1947, Art. 119. 
thermal behaviour of buildings. Broadly, what is required may be either $(i)$ a prediction of the transient behaviour of a given wall under given conditions, or (ii) a comparison of the transient behaviours of two different walls.

The first object of this paper is to discuss, in detail and from both the above points of view, one of the commonest transient problems, namely, that of finding the total quantity of heat which passes through unit area of a wall from zero time up to time $t$, the wall being initially at zero temperature and its surfaces being maintained at temperatures $V$ (constant), and zero, for times $t>0$. As remarked above, the complete calculation of this quantity would be extremely laborious, nevertheless it will be found possible by a simple arithmetical calculation to determine accurately a quantity characteristic of the wall, which we shall call the time-lag in establishing the steady state, which gives sufficient information for many purposes.

To illustrate what will be done, we consider first the case of a wall consisting of a single layer of thickness $l$, conductivity $k$, density $\rho$, specific heat $c$, and diffusivity $\alpha=k / \rho c$. Suppose that the wall is initially at zero temperature, and that for $t>0$ its faces $x=0$ and $x=l$ are maintained at constant temperatures, $V$ and zero, respectively. Then $Q_{0}$ and $Q_{l}$, the quantities of heat which cross unit area of the faces $x=0$ and $x=l$, respectively, up to time $t$, are given by $^{2}$

$$
\begin{aligned}
& Q_{n}=\frac{k V}{l}\left(t+\frac{l^{2}}{3 \alpha}\right)-\frac{2 k V l}{\alpha \pi^{2}} \sum_{n=1}^{\infty} \frac{1}{n^{2}} \exp \left(-\alpha n^{2} \pi^{2} t / l^{2}\right), \\
& Q_{l}=\frac{k V}{l}\left(t-\frac{l^{2}}{6 \alpha}\right)-\frac{2 k V l}{\alpha \pi^{2}} \sum_{n=1}^{\infty} \frac{(-1)^{n}}{n^{2}} \exp \left(-\alpha n^{2} \pi^{2} t / l^{2}\right) .
\end{aligned}
$$

For large values of the time the exponential terms in (2) and (3) tend to zero, and $Q_{0}$ and $Q_{l}$ tend to straight lines of slope $k V / l$ with intercepts on the time-axis of $\left(-l^{2} / 3 \alpha\right)$ and $\left(l^{2} / 6 \alpha\right)$ respectively. The way in which these linear asymptotes are approached is shown in Fig. 1 in which $Q_{0} / V l_{\rho c}$ and $Q_{l} / V l_{\rho c}$ are plotted against the dimensionless quantity $\alpha t / l^{2}$. It appears that the curves approach their asymptotes rapidly, and that at times of the order of the greater intercept, namely $l^{2} / 3 \alpha$, the departure from the asymptotes is quite small. Thus for times greater than this, the values of $Q_{0}$ and $Q_{l}$ are the values for steady flow for times $t-\tau_{0}$ and $t-\tau_{l}$, where $\tau_{0}=-l^{2} / 3 \alpha$ and $\tau_{l}=l^{2} / 6 \alpha$ may be described as the time-lags in establishing the steady state.

For the composite wall of $n$ layers, the complete solution for the quantity of heat crossing either surface up to time $t$ will have the form

$$
Q=V\left\{R_{1}+\cdots R_{n}\right\}^{-1}(t-\tau)+\text { negative exponentials, }
$$

and for large values of the time will be a straight line of slope given by (1) with intercept $\tau$ on the time axis. In Secs. 2 to 4 formulae will be derived for calculating the time-lag $\tau$ and it will be shown that the graph of $Q$ tends to its asymptote in much the same way as those of Fig. 1 so that a qualitative idea of the behaviour of $Q$ for times less than $\tau$ can be obtained.

The same method can be applied to any other problem in which the quantity under discussion ultimately increases linearly: solutions of a number of such problems are given in Sec. 5, they include cases of constant flux at a surface, linearly increasing tem-

${ }^{2}$ Barrer, Trans. Faraday Soc., 35, 628 (1939). Carslaw and Jaeger, loc. cit., Art. 117. 
perature at a surface, etc. In all these cases a time-lag appears which can be expressed in terms of the parameters defined in Secs. 3, 4. The behaviour of an unsymmetrical wall for opposite directions of flow is discussed in Sec. 6 .

In Sec. 7 the formulae derived in the earlier sections are collected for reference and a typical calculation set out in detail. It may be remarked here that the formulae are expressed in terms of the thermal resistances and capacities of the layers, so that they may be applied immediately to walls containing air gaps or films of finite thermal resistance and zero heat capacity.

The time-lags so obtained, being accurately calculable quantities which appear naturally in the solution of transient problems on composite walls, are also available for the comparison of the transient behaviour of different walls: it is suggested in Sec. 7 that the time lag in the quantity of heat passing through a wall when the temperature of its outer surface is suddenly changed gives a satisfactory criterion for such comparison.

2. The composite slab of $n$ layers with perfect thermal contact between them. Surface temperatures constant. Suppose the $r$-th layer has thickness $l_{r}$, conductivity $k_{r}$, specific heat $c_{r}$, density $\rho_{r}$, and diffusivity $\alpha_{r}=k_{r} / \rho_{r} c_{r}$. We suppose the layers to be the regions $0<x<l_{1}, l_{1}<x<l_{1}+l_{2}, \cdots$, etc., and in this section and section 4 that the outside surfaces, $x=0$ and $x=l_{1}+\cdots+l_{n}$, are kept at temperatures $V$ (constant) and zero, respectively, for $t>0$; the initial temperature of the whole being zero. As remarked in Sec. 1 we wish to find the quantities of heat $Q_{0}$ and $Q_{n}$ which cross unit area of the surfaces $x=0$ and $x=l_{1}+\cdots+l_{n}$ up to time $t$.

Let $v_{r}$ be the temperature in the $r$-th layer, and write $\bar{v}_{r}$ for its Laplace transform, that is

$$
\bar{v}_{r}=\int_{0}^{\infty} e^{-p t} v d t
$$

Then, writing

$$
q_{r}=\left(p / \alpha_{r}\right)^{1 / 2}, \quad r=1, \cdots, n,
$$

it is found by the usual Laplace transformation precedure ${ }^{3}$ that

$$
\begin{gathered}
\bar{v}_{1}=C_{1} \sinh q_{1} x+D_{1} \cosh q_{1} x, \quad 0<x<l_{1} \\
\bar{v}_{r}=C_{r} \sinh q_{r}\left(x-l_{1}-\cdots-l_{r-1}\right)+D_{r} \cosh q_{r}\left(x-l_{1} \cdots-l_{r-1}\right), \\
l_{1}+\cdots+l_{r-1}<x<l_{1}+\cdots+l_{r} .
\end{gathered}
$$

The $2 n$ constants $C_{1}, \cdots, D_{n}$ are to be determined from the conditions at the boundaries of the layers, namely,

$$
\begin{aligned}
\bar{v}_{1} & =V / p, & x & =0, \\
\bar{v}_{r} & =\bar{v}_{r+1}, & x & =l_{1}+l_{2}+\cdots+l_{r}, \\
k_{r} \frac{d \bar{v}_{r}}{d x} & =k_{r+1} \frac{d \bar{v}_{r+1}}{d x}, & x & =l_{1}+l_{2}+\cdots+l_{r}, \\
\bar{v}_{n} & =0, & x & =l_{1}+\cdots+l_{n} .
\end{aligned}
$$

${ }^{3}$ Cf. Carslaw and Jaeger, loc. cit., Art. 114. 
Using the abbreviations

$$
\begin{array}{ll}
\xi_{r}=q_{r} l_{r}, & r=1, \cdots, n, \\
\sigma_{r}=\frac{k_{r+1} \alpha_{r}^{1 / 2}}{k_{r} \alpha_{r+1}^{1 / 2}}, & r=1, \cdots, n-1,
\end{array}
$$

the $2 n$ equations (7) to (9) become

$$
\begin{aligned}
D_{1}=V / p & \\
C_{r} \sinh \xi_{r}+D_{r} \cosh \xi_{r}-D_{r+1}=0, & r=1, \cdots, n-1 \\
C_{r} \cosh \xi_{r}+D_{r} \sinh \xi_{r}-\sigma_{r} C_{r+1}=0, & r=1, \cdots, n-1 \\
C_{n} \sinh \xi_{n}+D_{n} \cosh \xi_{n}=0 . &
\end{aligned}
$$

In the next section we derive some properties of the determinants which appear in the solution of these equations, and in Sec. 4 the solution of the present problem is completed.

3. Properties of the determinants. Let $\Delta_{r}$ be the determinant of $2 r$ rows and columns defined by

$$
\left.\mid \begin{array}{cccccccc}
0 & 1 & 0 & 0 & 0 & \ldots & & \\
\sinh \xi_{1} & \cosh \xi_{1} & 0 & -1 & 0 & \ldots & & \\
\cosh \xi_{1} & \sinh \xi_{1} & -\sigma_{1} & 0 & 0 & \ldots & & \\
0 & 0 & \sinh \xi_{2} & \cosh \xi_{2} & 0 & -1 & \ldots & \\
0 & 0 & \cosh \xi_{2} & \sinh \xi_{2} & -\sigma_{2} & 0 & \ldots & \\
\ldots & \ldots & \ldots & \ldots & \ldots & \ldots & \ldots & \ldots
\end{array}\right]
$$

so that $\Delta_{n}$ is the determinant of the system of equations (12). Also let $\Omega_{r}$ be the determinant obtained from $\Delta_{r}$ by interchanging $\sinh \xi_{r}$ and $\cosh \xi_{r}$ in the last row. Finally let $\delta_{r}$ and $\omega_{r}$ be the determinants obtained from $\Delta_{r}$ and $\Omega_{r}$, respectively, by interchanging 0 and 1 in the first two columns of the first row.

All the solutions in which we are interested can be expressed in terms of the four determinants $\Delta_{n}, \Omega_{n}, \delta_{n}$, and $\omega_{n}$. To evaluate these we proceed as follows: expanding $\Delta_{r}$ and $\Omega_{r}$ by Laplace's development in terms of the last two columns we find 


$$
\left.\begin{array}{l}
\Delta_{r}=-\Delta_{r-1} \sigma_{r-1} \cosh \xi_{r}-\Omega_{r-1} \sinh \xi_{r} \\
\Omega_{r}=-\Delta_{r-1} \sigma_{r-1} \sinh \xi_{r}-\Omega_{r-1} \cosh \xi_{r},
\end{array}\right\}
$$

also

$$
\Delta_{1}=-\sinh \xi_{1}, \quad \Omega_{1}=-\cosh \xi_{1},
$$

and so $\Delta_{r}$ and $\Omega_{r}$ can be written down by repeated application of (14). For example,

$$
\begin{aligned}
\Delta_{2}= & \sigma_{1} \cosh \xi_{2} \sinh \xi_{1}+\sinh \xi_{2} \cosh \xi_{1}, \\
\Omega_{2}= & \sigma_{1} \sinh \xi_{2} \sinh \xi_{1}+\cosh \xi_{2} \cosh \xi_{1}, \\
\Delta_{3}= & -\sigma_{1} \sigma_{2} \cosh \xi_{3} \cosh \xi_{2} \sinh \xi_{1}-\sigma_{2} \cosh \xi_{3} \sinh \xi_{2} \cosh \dot{1}_{1} \\
& -\sigma_{1} \sinh \xi_{3} \sinh \xi_{2} \sinh \xi_{1}-\sinh \xi_{3} \cosh \xi_{2} \cosh \xi_{1} .
\end{aligned}
$$

Clearly, from their definitions, $\delta_{r}$ and $\omega_{r}$ satisfy the same recurrence relations (14) as $\Delta_{r}$ and $\Omega_{r}$, but in place of (15) we have

$$
\delta_{1}=\cosh \xi_{1}, \quad \omega_{1}=\sinh \xi_{1} .
$$

To obtain the results we need, the explicit expressions for these determinants are not required, but only the first two terms of their expansions in ascending powers of $p$. Writing

so that by (10)

$$
\xi_{r}=\eta_{r} p^{1 / 2}
$$

we find from (15)

$$
\eta_{r}=l_{r} \alpha_{r}^{-1 / 2}, \quad r=1, \cdots, n,
$$

$$
\left.\begin{array}{l}
\Delta_{1}=-\eta_{1} p^{1 / 2}\left(1+\frac{1}{6} \eta_{1}^{2} p+\cdots\right) \\
\Omega_{1}=-\left(1+\frac{1}{2} \eta_{1}^{2} p+\cdots\right) .
\end{array}\right\}
$$

Ẃ now assume

$$
\left.\begin{array}{l}
\Delta_{r}=(-1)^{r} \eta_{r} p^{1 / 2}\left(A_{r}+B_{r} p+\cdots\right) \\
\Omega_{r}=(-1)^{r}\left(A_{r}^{\prime}+B_{r}^{\prime} p+\cdots\right),
\end{array}\right\}
$$

substitute these values in the recurrence relations (14), and compare coefficients. This gives

$$
\begin{aligned}
\eta_{r} A_{r} & =\eta_{r-1} \sigma_{r-1} A_{r-1}+\eta_{r} A_{r-1}^{\prime} \\
\eta_{r} B_{r} & =\eta_{r-1} \sigma_{r-1} B_{r-1}+\frac{1}{2} \eta_{r}^{2} \eta_{r-1} \sigma_{r-1} A_{r-1}+\eta_{r} B_{r-1}^{\prime}+\frac{1}{6} \eta_{r}^{3} A_{r-1}^{\prime} \\
A_{r}^{\prime} & =A_{r-1}^{\prime} \\
B_{r}^{\prime} & =\sigma_{r-1} \eta_{r} \eta_{r-1} A_{r-1}+B_{r-1}^{\prime}+\frac{1}{2} \eta_{r}^{2} A_{r-1}^{\prime} .
\end{aligned}
$$


This chain of equations is to be solved successively starting with the values given by (20), viz.

$$
A_{1}=1, \quad B_{1}=\frac{1}{6} \eta_{1}^{2}, \quad A_{1}^{\prime}=1, \quad B_{1}^{\prime}=\frac{1}{2} \eta_{1}^{2} .
$$

The results may be simplified a little by introducing the quantities

$$
R_{r}=\frac{l_{r}}{k_{r}}, \quad R_{r}^{\prime}=\sum_{s=1}^{r} R_{s}, \quad H_{r}=l_{r} \rho_{r} c_{r}, \quad H_{r}^{\prime}=\sum_{s=1}^{r} H_{s},
$$

which have important physical interpretations: $R_{r}$ and $H_{r}$ are the thermal resistance and heat capacity, respectively, of unit area of the $r$-th layer, and $R_{r}^{\prime}$ and $H_{r}^{\prime}$ are the corresponding quantities for the first $r$ layers taken together. Using the notation (24) we get finally

$$
\begin{aligned}
& A_{r}=R_{r}^{\prime} / R_{r}, \\
& A_{r}^{\prime}=1, \\
& B_{r}=\left(\dot{R}_{r-1} / R_{r}\right) B_{r-1}+\frac{1}{2} R_{r-1}^{\prime} H_{r}+\frac{1}{6} R_{r} H_{r}+B_{r-1}^{\prime}, \\
& B_{r}^{\prime}=R_{r-1}^{\prime} H_{r}+\frac{1}{2} R_{r} H_{r}+B_{r-1}^{\prime} .
\end{aligned}
$$

(27) and (28) are to be solved successively, beginning with $B_{1}=(1 / 6) R_{1} H_{1}, B_{1}^{\prime}=$ $(1 / 2) R_{1} H_{1}$. The solution of (28) can be written down immediately, it is

$$
B_{r}^{\prime}=\frac{1}{2} \sum_{s=1}^{r} R_{s} H_{s}+\sum_{s=1}^{r-1} R_{s}^{\prime} H_{s+1},
$$

but there does not seem to be a simple expression for $B_{r}$.

In the same way, to determine the first terms of the expansions of $\delta_{r}$ and $\omega_{r}$. we assume

$$
\left.\begin{array}{l}
\delta_{r}=(-1)^{r-1} \frac{\left(k_{r} \rho_{r} c_{r}\right)^{1 / 2}}{\left(k_{1} \rho_{1} c_{1}\right)^{1 / 2}}\left(a_{r}+b_{r} p+\cdots\right) \\
\omega_{r}=(-1)^{r-1} \frac{p^{1 / 2}}{\left(k_{1} \rho_{1} c_{1}\right)^{1 / 2}}\left(a_{r}^{\prime}+b_{r}^{\prime} p+\cdots\right)
\end{array}\right\}
$$

and we find

$$
\begin{aligned}
& a_{r}=1, \\
& a_{r}^{\prime}=H_{r}^{\prime},
\end{aligned}
$$

and $b_{r}$ and $b_{r}^{\prime}$ are given by

$$
b_{r}=b_{r-1}+\frac{1}{2} R_{r} H_{r}+R_{r} H_{r-1}^{\prime}
$$




$$
b_{r}^{\prime}=b_{r-1}^{\prime}+b_{r-1} H_{r}+\frac{1}{6} R_{r} H_{r}^{2}+\frac{1}{2} R_{r} H_{r} H_{r-1}^{\prime}
$$

with

$$
b_{1}=\frac{1}{2} R_{1} H_{1}, \quad b_{1}^{\prime}=\frac{1}{6} R_{1} H_{1}^{2}
$$

It follows immediately from (33) that

$$
b_{r}=\frac{1}{2} \sum_{s=1}^{r} R_{s} H_{s}+\sum_{s=1}^{r-1} R_{s+1} H_{s}^{\prime} .
$$

4. Solution of the problem of Section 2. The solution of the equations (12) can now be written down in terms of the determinants of Sec. 3 . We shall require $C_{1}, C_{n}$, and $D_{n}$. It follows immediately that

$$
C_{1}=\frac{V \delta_{n}}{p \Delta_{n}}
$$

Also, evaluating the determinants which occur in the numerators of $C_{n}$ and $D_{n}$ by Laplace's expansion in terms of their last two columns, we find

$$
C_{n}=(-1)^{n-1} \frac{V \cosh \xi_{n}}{p \Delta_{n}}, \quad D_{n}=(-1)^{n} \frac{V \sinh \xi_{n}}{p \Delta_{n}} .
$$

We have to find $Q_{0}$, the total amount of heat which crosses unit area of the plane $x=0$ up to time $t$, and $Q_{n}$, the total amount crossing unit area of the plane $x=l_{1}+$ $\cdots+l_{n}$ up to time $t$.

$Q_{0}$ is given by

$$
-k_{1} \int_{0}^{t}\left[\frac{\partial v_{1}}{\partial x}\right]_{x=0} d t
$$

and its Laplace transform is

$$
\bar{Q}_{0}=-\frac{k_{1}}{p}\left[\frac{d \bar{v}_{1}}{d x}\right]_{x=0}=-\frac{k_{1} q_{1} C_{1}}{p} .
$$

Therefore, using (37),

$$
\bar{Q}_{0}=-\frac{k_{1} q_{1} V \delta_{n}}{p^{2} \Delta_{n}}
$$

Similarly, using (38),

$$
\bar{Q}_{n}=(-1)^{n} \frac{k_{n} V q_{n}}{p^{2} \Delta_{n}}
$$

$Q_{0}$ and $Q_{n}$ are found from their Laplace transforms by the use of the inversion theorem followed by contour integration. Thus

$$
Q_{0}=\frac{1}{2 \pi i} \int_{\gamma-i \infty}^{\gamma+i \infty} e^{p t} \bar{Q}_{0}(p) d p, \quad R(\gamma)>0 .
$$

The integrand has an infinite number of poles on the negative real axis which give rise to terms involving negative exponentials in the time, also it has a double pole at 
the origin which gives the asymptote we require. To find the residue at the origin the expansions of $\delta_{n}$ and $\Delta_{n}$ developed in Sec. 3 are needed. Using (21) and (30) we get

$$
\bar{Q}_{0} e^{p t}=\frac{V\left(a_{n}+b_{n} p+\cdots\right)}{R_{n} p^{2}\left(A_{n}+B_{n} p+\cdots\right)} e^{p t} .
$$

Therefore the contribution of the pole at the origin to $Q_{0}$ is

$$
Q_{0}=\frac{V}{R_{n}^{\prime}}\left\{t-\left(\frac{B_{n}}{A_{n}}-b_{n}\right)\right\}
$$

where the values (25) and (31) of $A_{n}$ and $a_{n}$ have been used. $R_{n}^{\prime}$ is, of course, the thermal resistance of the whole wall so that (42) corresponds to steady flow for time

$$
t-\left(\frac{B_{n}}{A_{n}}-b_{n}\right) \text {. }
$$

In the same way it follows from (40) that for large values of the time

$$
Q_{n}=\frac{V}{R_{n}^{\prime}}\left(t-\frac{B_{n}}{A_{n}}\right) \text {. }
$$

Thus the time lags at the first and last surfaces are respectively

$$
\frac{B_{n}}{A_{n}}-b_{n} \quad \text { and } \quad \frac{B_{n}}{A_{n}} .
$$

These are calculated from the formulae of Sec. 3: numerical examples of the procedure are given in Sec. 7 .

It remains to discuss the way in which the linear asymptotes (42) and (43) are approached. For a complete solution it is necessary to find the zeros of $\Delta_{n}$, qua function of $p$, from which the exponential terms in the complete solution are obtained. Now from its definition $\left(-A_{n} / B_{n}\right)$ is a crude first approximation to the smallest zero of $\Delta_{n}$, and, moreover, one which is certainly too small in magnitude. It follows at once that the departures of the curves for $Q_{0}$ and $Q_{n}$ from their asymptotes have an exponential factor which decreases more rapidly than

$$
\exp \left(-A_{n} t / B_{n}\right) .
$$

In the statement of Sec. 2 there was supposed to be perfect thermal contact at the surfaces of the layers, and the two outside surfaces were supposed to be at temperatures $V$ and zero. Normally there are air gaps or films or contact resistances of some sort at the outside surfaces and between the layers: these may be taken into account by regarding each contact resistance as an additional layer of the appropriate thermal resistance and zero heat capacity.

If the wall is initially at temperature $V$ instead of zero, $Q_{0}$ and $Q_{n}$ have the same values as those for the wall initially at zero with $x=0$ maintained at zero and $x=$ $l_{1}+\cdots+l_{n}$ at $-V$ for $t>0$. Thus the time lags at the two surfaces are those, obtained as above, for flow through the wall in the reverse direction. The calculation of these is discussed in Sec. 6 .

Finally, it may be remarked that, since explicit expressions for $\Delta_{n}$ and $\delta_{n}$ such as (16) can be written down from (14), explicit formulae for the Laplace transforms of $Q_{0}$ and $Q_{n}$ and of the temperatures in the slab can also be written down. The solutions 
for harmonic surface temperature also follow in the usual way from these Laplace transforms.

5. Other problems on the composite slab. The solutions of a number of other problems on the composite slab behave in the same way as $Q_{0}$ and $Q_{n}$ of Sec. 4 , and in all cases the time lags can be expressed in terms of the eight quantities $A_{n}, \cdots, b_{n}^{\prime}$ defined in Sec. 3.

In all the cases below, the slab and notation are those of Sec. 2 and the slab is initially at zero temperature; only the boundary conditions at the surfaces are changed.

(i) Constant flux $F$ at $x=0$ for $t>0$. Zero temperature at $x=l_{1}+\cdots+l_{n}$.

In this case the quantity of heat $Q_{n}$ which has crossed unit area of the plane $x=l_{1}+$ $\cdots+l_{n}$ up to time $t$ is ultimately

$$
Q_{n}=F\left(t-b_{n}\right) .
$$

(ii) Constant flux $F$ at $x=0$ for $t>0$. No flow of heat at $x=l_{1}+\cdots+l_{n}$.

Here the temperature at $x=0$ is ultimately

$$
\frac{F}{H_{n}^{\prime}}\left\{t-\frac{b_{n}^{\prime}}{a_{n}^{\prime}}+B_{n}^{\prime}\right\}
$$

and the temperature at $x=l_{1}+\cdots+l_{n}$ is ultimately

$$
\frac{F}{H_{n}^{\prime}}\left\{t-\frac{b_{n}^{\prime}}{a_{n}^{\prime}}\right\} \text {. }
$$

(iii) Linearly increasing temperature Vt at $x=0$, and no flow of heat at $x=l_{1}+\cdots+l_{n}$. The temperature at $x=l_{1}+\cdots+l_{n}$ is ultimately

$$
V\left\{t-B_{n}^{\prime}\right\}
$$

6. Flow through the same wall in opposite directions. Most composite walls are not symmetrical, so that, though their thermal resistance is independent of the direction of flow of heat, their transient behaviour may be expected to be different for the two directions. It is also necessary to study flow in the reverse direction for the problem mentioned at the end of Sec. 4 .

Considering again the wall of Sec. 2, suppose now that the surface $x=l_{1}+\cdots+l_{n}$ is maintained at $V$ for $t>0$ and the surface $x=0$ at zero, the initial temperature of the wall being zero. The only modification is that the first and last of equations (12) are replaced by

$$
\left.\begin{array}{cc}
D_{1} & =0 \\
C_{n} \sinh \xi_{n}+D_{n} \cosh \xi_{n} & =V / p
\end{array}\right\}
$$

If $Q_{0}$ is the flow per unit area from right to left across the surface $x=0$ up to time $t$, and $Q_{n}$ that across the surface $x=l_{1}+\cdots+l_{n}$, we find on solving that

$$
\begin{aligned}
& \bar{Q}_{0}=(-1)^{n} \frac{V k_{n} q_{n}}{p^{2} \Delta_{n}}, \\
& \bar{Q}_{n}=\frac{V k_{n} q_{n} \Omega_{n}}{p^{2} \Delta_{n}} .
\end{aligned}
$$


Since (50) is identical with (40) it follows that the quantity of heat passing through the wall up to time $t$ is independent of the direction of flow. This is a special case of a general reciprocal theorem.

The quantities of heat passing into the wall, of course, are different, but a simple result about the time lags follows at once. From (51), in the usual way, we find that for large values of the time

$$
Q_{n}=\frac{V}{R_{n}^{\prime}}\left\{t-\left(\frac{B_{n}}{A_{n}}-B_{n}^{\prime}\right)\right\}
$$

Comparing this with the corresponding quantity (42) it appears that $b_{n}$ has been replaced by $B_{n}^{\prime}$.

These results, so far as time-lags are concerned, may be summarized by the statement that for flow in the reverse direction in a given wall with zero initial temperature, $B_{n} / A_{n}$ and $b_{n}^{\prime} / a_{n}^{\prime}$ are unaltered, while $b_{n}$ and $B_{n}^{\prime}$ are interchanged. The latter remark follows also from the formulae (36) and (29). It may also be remarked here that $b_{n}$ is the time taken for a quantity of heat equal to the steady state heat content of the wall with constant and zero surface temperatures to flow through the wall under these conditions, while $B_{n}^{\prime}$ is the corresponding quantity for flow in the reverse direction.

7. Numerical calculations for a typical wall and discussion of results. First we collect for reference the formulae derived earlier. The fundamental thermal quantities for the layers are

$$
R_{r}=\frac{l_{r}}{k_{r}}, \quad R_{r}^{\prime}=\sum_{s=1}^{r} R_{s}, \quad H_{r}=l_{r} \rho_{r} c_{r}, \quad H_{r}^{\prime}=\sum_{s=1}^{r} H_{s} .
$$

The time lags of $\S \S 4,5$ all involved the four quantities

$$
B_{n} / A_{n}, \quad B_{n}^{\prime}, \quad b_{n}, \quad b_{n}^{\prime} / a_{n}^{\prime},
$$

where

$$
\begin{aligned}
& A_{r}=R_{r}^{\prime} / R_{r}, \quad a_{r}^{\prime}=H_{r}^{\prime} \\
& B_{1}=\frac{1}{6} R_{1} H_{1}, \quad B_{1}^{\prime}=\frac{1}{2} R_{1} H_{1}, \quad b_{1}=\frac{1}{2} R_{1} H_{1}, \quad b_{1}^{\prime}=\frac{1}{6} R_{1} H_{1}^{2} \\
& B_{r}=\left(R_{r-1} / R_{r}\right) B_{r-1}+\frac{1}{2} R_{r-1}^{\prime} H_{r}+\frac{1}{6} R_{r} H_{r}+B_{r-1}^{\prime} \\
& B_{r}^{\prime}=R_{r-1}^{\prime} H_{r}+\frac{1}{2} R_{r} H_{r}+B_{r-1}^{\prime} \\
& b_{r}=b_{r-1}+\frac{1}{2} R_{r} H_{r}+R_{r} H_{r-1}^{\prime} \\
& b_{r}^{\prime}=b_{r-1}^{\prime}+b_{r-1} H_{r}+\frac{1}{6} R_{r} H_{r}^{2}+\frac{1}{2} R_{r} H_{r} H_{r-1}^{\prime} .
\end{aligned}
$$

We first study in detail the wall described in Table I: units are lbs., ft., hrs., B.T.U., and ${ }^{\circ} \mathrm{F}$. 
TABLE I.

\begin{tabular}{c|l|l|l|l|l|l}
\hline \hline Layer & Substance & $l$ & $\rho c$ & $k$ & $H_{r}$ & $R_{r}$ \\
\cline { 2 - 4 } 1 & Air film & & & & 0 & 0.25 \\
2 & Brick & 0.333 & 25 & 0.42 & 8.33 & 0.79 \\
3 & Cavity & & & & 0 & 0.75 \\
4 & Brick & 0.333 & 25 & 0.42 & 8.33 & 0.79 \\
5 & Plaster & 0.083 & 15 & 0.25 & 1.25 & 0.33 \\
6 & Air film & & & & 0 & 0.60 \\
\hline
\end{tabular}

For numerical calculations it is most convenient to fill in the thermal quantities $H_{r}$ and $R_{r}$ in a table such as Table II, then to calculate the subsidiary quantities such as $H_{r}^{\prime}, \frac{1}{2} R_{r} H_{r}$, etc. from them, and finally to work down the columns for $B_{r}, B_{r}^{\prime}, b_{r}, b_{r}^{\prime}$ using (56) to (59). If only a single time lag is needed, all these columns need not be filled in.

TABLE II.

\begin{tabular}{|c|c|c|c|c|c|c|c|c|c|c|c|c|c|c|}
\hline 灾 & $H_{r}$ & $H_{r}^{\prime}$ & $R_{r}$ & $R_{r}^{\prime}$ & $\frac{R_{r-1}}{R_{r}} R_{r}^{\prime}$ & ${ }_{-1} H_{r}$ & $H_{r-1}^{\prime}$ & $\frac{1}{2} R_{r} H_{r}$ & $A_{r}$ & $B_{r}$ & $B_{r}^{\prime}$ & $b_{r}$ & $a_{r}^{\prime}$ & $b_{r}^{\prime}$ \\
\hline 1 & 0 & 0 & 0.25 & 0.25 & & & & 0 & 1 & 0 & 0 & 0 & 0 & 0 \\
\hline 2 & 8.33 & 8.33 & 0.79 & 1.04 & 0.32 & 2.08 & 0 & 3.29 & 1.32 & 2.14 & 5.37 & 3.29 & 8.33 & 9.13 \\
\hline 3 & 0 & 8.33 & 0.75 & 1.79 & 1.05 & 0 & 6.25 & 0 & 2.39 & 7.62 & 5.37 & 9.54 & 8.33 & 9.13 \\
\hline 4 & 8.33 & 16.66 & 0.79 & 2.58 & 0.95 & 14.91 & 6.58 & 3.29 & 3.27 & 21.16 & 23.57 & 19.41 & 16.66 & 125.1 \\
\hline 5 & 1.25 & 17.91 & 0.33 & 2.91 & 2.39 & 3.22 & 5.50 & 0.21 & 8.82 & 75.91 & 27.00 & 25.11 & 17.91 & 152.9 \\
\hline 6 & 0 & 17.91 & 0.60 & 3.51 & 0.55 & 0 & 10.75 & 0 & 5.85 & 68.75 & 27.00 & 35.86 & 17.91 & 152.9 \\
\hline
\end{tabular}

The formulae and results for the time lags in the problem of $\$ \S 2-4$ and in those of Sec. 5 are summarized in Table III where the corresponding formulae (55) for a single layer of resistance $R$ and heat capacity $H$ are also given for subsequent reference. The results for the wall described in Table I are given under "Wall 1". "Wall 2" differs from Wall 1 only in that the layers of brick are replaced by layers of wood of thickness 0.066 , $\rho c=10, k=0.083, H_{r}=0.66, R_{r}=0.79$. Wall 2 thus has the same overall thermal resistance $R_{6}^{\prime}=3.51$ as Wall 1 , but its overall heat capacity $H_{6}^{\prime}$ is 2.57 in place of 17.91 .

Walls 1 and 2 are idealized building walls of brick and timber construction respectively. The flow through these up to time $t$ (hours) per unit temperature difference between the surfaces is, for large $t$,

$$
\frac{1}{3.51}(t-11.8) \quad \text { and } \quad \frac{1}{3.51}(t-1.6)
$$

B.T.U. respectively, showing clearly the important effect of the difference in heat capacity of the two walls.

This investigation was begun in connection with the flow of heat in refrigerated rail cars.* These are cooled by ice carried inside, and the quantity $Q_{n}$ of Sec. 6 determines

${ }^{*}$ I am indebted to Mr. E. W. Hicks of the Division of Food Preservation and Transport, Council for Scientific and Industrial Research, Australia, for suggesting the problem and much useful discussion. 
TABLE III.

\begin{tabular}{|c|c|c|c|c|c|c|}
\hline Problem & $\begin{array}{l}\text { Boundary } \\
\text { conditions }\end{array}$ & Quantity & Time lag & $\begin{array}{l}\text { Single } \\
\text { layer }\end{array}$ & Wall 1 & Wall 2 \\
\hline \multirow[t]{2}{*}{ Sec. 2} & & & $\left(B_{n} / A_{n}\right)-b_{n}$ & $-\frac{1}{3} R H$ & -24.11 & -2.13 \\
\hline & Zero Temp. & $Q_{n}$ & $B_{n} / A_{n}$ & $\frac{1}{6} R H$ & 11.75 & 1.59 \\
\hline \multirow[t]{2}{*}{ Sec. $5(i)$} & Const. Flux & & & & & \\
\hline & Zero Temp. & $Q_{n}$ & $b_{n}$ & $\frac{1}{2} R H$ & 35.86 & 3.72 \\
\hline \multirow[t]{2}{*}{ Sec. 5(ii) } & Const. Flux & $v_{0}$ & $\left(b_{n}^{\prime} / a_{n}^{\prime}\right)-B_{n}^{\prime}$ & $-\frac{1}{3} R H$ & -18.46 & 4.11 \\
\hline & Zero Flux & $v_{n}$ & $b_{n}^{\prime} / a_{n}^{\prime}$ & $\frac{1}{6} R H$ & 8.54 & 1.19 \\
\hline \multirow[t]{2}{*}{ Sec. 5(iii) } & Linear Temp. & & & & & \\
\hline & Zero Flux & $v_{n}$ & $B_{n}^{\prime}$ & $\frac{1}{2} R H$ & 27.00 & 5.30 \\
\hline
\end{tabular}

the quantity of ice melted up to time $t$. The time lag in this quantity is $B_{n} / A_{n}-B_{n}^{\prime}$, a knowledge of this provides sufficient information to decide on the performance of a car in practice. A discussion of the magnitude of this time lag for various car designs will be given elsewhere.

Here it is desired to stress the general applicability of these time-lags for providing an easily calculated criterion for comparing the performance of different walls under a sudden change of external conditions. Any of the cases of Table III may be used, but the most generally useful is $B_{n} / A_{n}$, the time lag in transfer of heat through a wall when its outer temperature is suddenly raised.

Finally, the question of how far it is possible to define an "equivalent homogeneous wall" which will have the same transient behaviour as a given composite wall should be discussed. If the thermal resistance and heat capacity of this equivalent homogeneous wall are $R$ and $H$ it follows from Table III that we must have

$$
R H=\frac{6 B_{n}}{A_{n}}=2 b_{n}=2 B_{n}^{\prime}=\frac{6 b_{n}^{\prime}}{a_{n}^{\prime}} .
$$

In actual walls, the four quantities on the right of (60) are often far from equal; thus for Walls 1 and 2 of Table III they are respectively 70.5, 71.7, 54, 51.2 and 9.5, 7.4, 10.6, 7.1. Thus if an equivalent homogeneous wall is defined based on any particular transient phenomenon it may be expected to be a good deal out in the estimates of others. 